eases.” Dr. Martone also is president-elect of the Society for Healthcare Epidemiology of America.

FROM: National Foundation for Infectious Diseases. NFID's new senior executive director. The Double Helix 1995;20(3):1.

\section{National Academy of Sciences Endorses Syringe Exchange Programs}

The National Academy of Sciences, a federally chartered independent research organization, recently reviewed research on syringe exchange programs and concluded that these programs should be regarded as an effective component of a comprehensive strategy to prevent infectious disease and that states should rescind laws restricting the sale and possession of syringes. Numerous studies have shown that syringe exchange programs and other interventions that increase access to sterile syringes can prevent infections, including HIV, hepatitis B, and hepatitis $\mathrm{C}$. These findings open the way for the Clinton Administration to lift a legal ban on federal financing of such programs.

Approximately 75 needle exchange programs operate in 55 cities in the United States, using private and other nonfederal financing. Many people fear that such syringe exchange programs would worsen drug abuse and encourage illegal activity. The academy's report said that the HIV epidemic in this country now clearly is driven by infection occurring in the population of injection drug users, their sexual partners, and their offspring. The report also said that the epidemiology of HIV was changing, with the proportion of new AIDS cases represented by gay men down to $47 \%$ in 1993 from $74 \%$ in 1981 , while new cases attributed to the use of illicit intravenous drugs rose to $28 \%$ in 1993 from $12 \%$ in 1981 .

Since the late 1980s, Congress has prohibited the use of federal money to support needle exchange programs. While there are many that support the academy's findings, there also is strong opposition. Dr. Lincoln Moses, chairman of the 15-member panel that prepared the report, said, "the federal government should not force such programs on those that oppose them, but rather remove existing restraints so people can make their own decisions."

Syringe exchange programs are relatively inexpensive, compared with other preventive efforts, because syringes cost less than 10 cents each, and many programs are staffed by volunteers. However, federal prohibitions make it difficult for public health officers to plan and execute compressive AIDS prevention strategies.
FROM: Needle exchanges endorsed as AIDS strategy. New York Times September 20, 1995;A:1.

Centers for Disease Control and Prevention. Syringe exchange programs-United States, 1994-1995. MMWR 1995;44(37):684-685,691.

\section{Bloodstream Infections With Needleless Devices}

Researchers from the CDC and the Children's Hospital Oakland, conducted a retrospective cohort study of pediatric oncology patients receiving home health care from January 1992 through November 1994. Case patients were those that presented from home with fever, with or without chills, and with more than one positive blood culture.

The only procedural change in these patients was the introduction of a needleless device for central venous catheter (CVC) access in May 1993. The home health agencies provided supplies and limited nursing care, while parents were responsible for maintaining and accessing the central lines at home following training during their child's hospitalization.

Of 185 patients with CVCs during the study period, 59 (32\%) contracted 91 bloodstream infections during 75,148 CVC days. All patients with bloodstream infections required hospitalization; none died. Gram-negative organisms were isolated more frequently than gram-positive organisms. Previously reported risk factors, including catheter type, patient age or gender, and diagnosis were not associated with increased bloodstream infection rates. After introduction of the needleless devices into the home healthcare regimen, bloodstream infection rates increased $80 \%$ (0.81 versus 1.46 bloodstream infections per 1,000 CVC days).

The data suggest that pediatric patients receiving home health care via needleless devices may be at increased risk for bloodstream infections.

FROM: Kellerman S, Shay D, Howard J, et al. Bloodstream infections associated with needleless devices used for central venous catheter access in children receiving home health care. Presented at the 35th Interscience Conference on Antimicrobial Agents and Chemotherapy, San Francisco, CA, September 17-20, 1995. Abstract J11.

Additional news items in this issue: Rifampin-Resistant, Isoniazid-Susceptible TB in HIV Patients, page 622. 